\title{
Alimentación de los peces dulceacuícolas tropicales Heterandria bimaculata y Poecilia sphenops (Cyprinidontiformes: Poeciliidae)
}

\author{
Patricia Trujillo-Jiménez \& Héctor Toledo Beto \\ Universidad Autónoma del Estado de Morelos, Centro de Investigaciones Biológicas, Av. Universidad 1001 Col. \\ Chamilpa C. P. 62210, Cuernavaca, Morelos, México. Tel. (01 777) 329-70-29, Fax (01 777) 329-70-56; \\ trujill@cib.uaem.mx.
}

Recibido 12-III-2004. Corregido 13-XI-2006. Aceptado 28-II-2007.

\begin{abstract}
Diet of the tropical freshwater fish Heterandria bimaculata (Haeckel) and Poecilia sphenops Valenciennes (Cyprinidontiformes: Poeciliidae). We analyzed the diet and feeding habits of the fishes Heterandria bimaculata and Poecilia sphenops. Specimens were captured monthly in "Los Carros" damp, Morelos, Mexico $\left(18^{\circ} 37^{\prime} \mathrm{N}, 98^{\circ} 43^{\prime} \mathrm{W}\right)$. We quantified gut content by the numerical method and by the frequency of occurrence method; and used the MacArthur and Levin's indices for niche overlap. The diet of $H$. bimaculata was composed by 16 prey categories, mainly dipterans (Culicidae predominated), independently of sex, size and season. The index of niche overlap was high, from 0.74 to 0.99 . The diet of $P$. sphenops consisted of 11 items, detritus being the most consumed, also independently of sex, size and season. The niche overlap index was high (0.99), indicating overlapping for all analyses. There was little diet overlap (0.26) between the two species. Rev. Biol. Trop. 55 (2): 603-615. Epub 2007 June, 29.
\end{abstract}

Key words: diet, food habits, Heterandria bimaculata, Poecilia sphenops, "Los Carros”, Morelos, Mexico.

La familia Poeciliidae, comprende peces nativos del Nuevo Mundo y de distribución principalmente tropical. Está constituida por alrededor de 190 especies, clasificadas en 22 géneros y 12 subgéneros. Se distribuyen en agua dulce, salobre y marina desde el noreste de los Estados Unidos hasta el sur del Río de la Plata en la parte norte de Argentina, con gran diversificación taxonómica en América Central, México y las Indias Occidentales (Alayo 1973, Meffe y Snelson 1989). Este elevado ámbito de adaptabilidad y tolerancia caracteriza al grupo como un excelente colonizador, con un amplio espectro de hábitos tróficos (Hess y Tarzwell 1942, Miley 1978, Greenfield et al. 1983a, Turner y Snelson 1984, Meffe y Snelson 1989, Parenti y Rauchenberger 1989). Pese al uso de los poecilidos como modelos en estudios genéticos, fisiológicos y médicos tiene ya muchos años de aplicación, es notable la falta de conocimientos sobre su biología y ecología, siendo pobre el número de investigaciones realizadas tanto en ambiente marino como en continental (Suárez et al. 2002).

Heterandria bimaculata "guatopote manchado" y Poecilia sphenops "moli o topote mexicano", son dos especies mexicanas, cuyas tallas no sobrepasan los $120 \mathrm{~mm}$ de longitud total. Ambas especies presentan una amplia distribución geográfica, habitan principalmente en zonas cercanas a las orillas de lagos, presas, ríos y manantiales, donde los machos adultos defienden sus territorios y las crías encuentran refugio entre la vegetación. Asimismo, estas especies son importantes en la acuariofilia en México. Actualmente en el estado de Morelos, México, estas especies ocupan lagos, ríos, manantiales y presas, cuyas poblaciones son abundantes, sin embargo, son pocos los estudios sobre su biología que se han realizado en condiciones naturales, por lo cual el propósito de este artículo es obtener información sobre su 
alimentación y hábitos alimentarios y la relación que guardan con el tracto digestivo.

\section{MATERIALES Y MÉTODOS}

La presente investigación se realizó en el embalse "Los Carros", el cual se localiza en el Municipio de Axochiapán, Morelos, México, a una altitud de $1100 \mathrm{msnm}$, a los $18^{\circ} 37^{\prime} \mathrm{N}$, 98 $43^{\circ} \mathrm{W}$, cerca del límite de los estados de Puebla y Morelos. Forma parte del sistema de riego "Los Carros-Cayehuacán", mismo que cuenta con $69.7 \mathrm{~km}$ de canales de riego distribuidos en ambos estados. Presenta un volumen aproximado de diez millones de metros cúbicos y profundidad máxima de $30 \mathrm{~m}$ (MagallónBarajas et al. 1992).

"Los Carros" se encuentra dentro de la provincia fisiográfica Lagos y Volcanes del Anáhuac, y recibe el aporte del río San Francisco, mismo que corresponde a la región hidrológica del río Balsas. El sustrato geológico se compone de rocas sedimentarias e ígneas; la vegetación es pastizal inducido de tipo xerófito con predominancia en Mimosa sp. y Opuntia sp. El clima de la región pertenece al tipo A Wo (W), que indica un clima cálido húmedo con lluvias en verano y un porcentaje de lluvia invernal menor de $5 \mathrm{~mm}$. La precipitación media anual fluctúa entre 800 y $1000 \mathrm{~mm}$, la temperatura media anual de $22.7^{\circ} \mathrm{C}$ (MagallónBarajas et al. 1992).

El trabajo comprendió un ciclo anual de muestreos mensuales, para cada muestreo se realizó la captura de peces mediante una red de $3 \mathrm{~m}$ de largo por $2 \mathrm{~m}$ de ancho, con una abertura de malla de $1.5 \mathrm{~cm}$. haciendo arrastres en las orillas recolecciónndo ambas especies al mismo tiempo. Los especímenes fueron colocados en frascos con formol al $10 \%$ previamente etiquetados. A cada ejemplar se les midió la longitud patrón (LP) en mm y se le determinó el sexo. Con un total de 20 ejemplares de cada especie se llevó a cabo la descripción del tracto digestivo (Moyle y Cech 1988).

El tracto digestivo de ambas especies se dividió en tres partes iguales, tomando el contenido del tercio medio para su análisis (Trujillo-Jiménez y Díaz-Pardo 1996). La identificación del contenido intestinal se realizó con un microscopio estereoscópico, tratando de llegar a la categoría taxonómica más cercana a la especie, basándose en los criterios de Needham y Needham (1978), Pennak (1978), Bland (1979), Chu (1979) y Merrit y Cummins (1996). Asimismo, se contó con la ayuda de especialistas.

Para la cuantificación del contenido intestinal se utilizó el método numérico por cuadrícula (No) y el de frecuencia de presencia (FO) (Lagler 1956, Windell y Bowen 1978). El análisis de la dieta se hizo global, por temporadas, sexos y tallas. Para conocer el traslape de nicho se empleó el índice de MacArthur y Levin, modificado por Pianka (Krebs 1999). Para corroborar los resultados de traslape de la dieta o las posibles diferencias de alimentación de las especies, se utilizó la prueba de $\chi^{2}$ (Siegel 1976).

\section{RESULTADOS}

\section{Tracto digestivo}

H. bimaculata presenta una boca en posición subterminal, ligeramente dirigida hacia el dorso, ambas mandíbulas presentan una hilera de dientes caninos y la superior es apenas protráctil. Tiene cuatro arcos branquiales, el primero con 22 a 24 branquiespinas, con dientes faríngeos cónicos dispuestos en dos placas superiores y dos inferiores, estos últimos son más pequeños y soportan un menor número, en toda la placa los dientes no muestran un arreglo uniforme (Álvarez del Villar 1970). El estómago está bien definido y el tubo digestivo es muy corto. Después del estómago se presenta una flexión hacia delante y casi inmediatamente se dobla nuevamente hacia atrás formando un semicírculo para desembocar en el ano. El tracto (de esófago a ano) ocupa el $30 \%$ de la cavidad visceral, el estómago tiene una dimensión igual a la quinta parte de la longitud patrón y la del intestino es aproximadamente la cuarta parte de la misma longitud. 
P. sphenops presenta boca subterminal, ligeramente dirigida hacia el dorso, posee un premaxilar con gran protactibilidad. El dentario es un hueso par de forma más o menos redondeada, alargado lateralmente y aplanado dorsoventralmente, formando junto a su par simétrico la totalidad de la mandíbula inferior por lo que en su mayor porción dorsal sostiene una amplia placa de dientes y en su porción anterior, una hilera de dientes largos y aplanados. Sobre el borde anterior del hueso, sobresale una hilera de 20 a 25 dientes, casi todos de igual tamaño excepto los laterales que son ligeramente más cortos, todos ellos proyectándose anteriormente en forma recta pero, en su último tercio, se curva ligeramente a nivel ventral y su ápice se aplana a manera de espátula. La cara dorsal del dentario tiene una placa de dientes extendida en toda su superficie formando un "racimo" de pequeñas piezas tricúspides ubicados irregularmente (Paredes 1998). Los dientes faríngeos se encuentran en dos placas triangulares, presentan una serie de dientes diferentes: espatulados y en forma de peine que se encuentran asociados con los dientes del premaxilar y dentario. Tiene cuatro arcos branquiales, cuyo número de branquiespinas va de 24 a 27 en el primer arco branquial. El tracto digestivo es indiferenciado, no hay estómago bien definido, el tracto tiene de siete a ocho pliegues formándose un espiral y es de 3.5 a 4.5 veces mayor que la longitud patrón y ocupa el $50 \%$ de la cavidad visceral.

\section{Análisis intraespecífico}

\section{H. bimaculata}

Composición global de la dieta: la dieta de H. bimaculata (190 ejemplares) está constituida por 16 componentes, registrándose una predilección mayor por los alimentos de origen animal, dado que su porcentaje de ingestión fue de $99.6 \%$ (92.9\% corresponde a insectos) y el de preferencia de $198.4 \%$. Dentro de los componentes alimenticios de origen animal, se observó al orden Diptera con los porcentajes más altos en ambos métodos del cual se determinaron dos familias (Simuliidae y Culicidae), siendo la familia Culicidae la más representativa. Los himenópteros fueron el segundo orden con porcentajes altos, seguidos por el orden Coleoptera, todos considerados como alimentos secundarios. Los peces (Micropterus salmoides) presentan un valor de ingestión de $4.1 \% \mathrm{y}$ de frecuencia $6.2 \%$. El porcentaje de ingestión indica que los peces alcanzaron una proporción relativamente alta debido a su tamaño con respecto a los otros componentes alimenticios. El resto de los componentes de origen animal exhibieron proporciones bajas. Dentro de los artículos de origen vegetal, únicamente se registraron semillas y restos vegetales con proporciones bajas, razón por la cual son alimentos circunstanciales (Cuadro 1).

Análisis temporal de la dieta: para cubrir este apartado se analizaron las dos épocas del año más marcadas dentro del estado de Morelos, lluvias (mayo a octubre) y estiaje (noviembre a abril), promediando los resultados mensuales de cada una de esas épocas. Como puede apreciarse en el cuadro 2 en las dos épocas, el alimento de origen animal se mostró notablemente mayor tanto en ingestión como en preferencia. En ambas épocas el orden Diptera fue el que presentó los mayores porcentajes, reconociéndose en las lluvias como alimento preferente y en el estiaje como alimento circunstancial, la familia Culicidae fue la más representativa, considerada por sus valores de preferencia como alimento circunstancial en ambas épocas. El orden Hymenoptera, fue el segundo más ingerido, exhibiendo porcentajes más altos en las lluvias, registrándose como alimento preferencial y en el estiaje como secundario. El orden Odonata exhibió porcentajes relativamente altos en las lluvias a diferencia del estiaje, no obstante, debido al porcentaje obtenido con el método de FO, en ambas temporadas son considerados como alimento circunstancial. Los coleópteros se comportaron a la inversa de los odonatos, registrando un porcentaje mayor en el estiaje considerados como alimento circunstancial. Es importante señalar que aunque los peces ( $M$. salmoides) 
CUADRO 1

Dieta general de H. bimaculata $y$ P. sphenops, de acuerdo con los métodos numérico (No) y Frecuencia de Presencia (F.O.)

TABLE 1

General diet of $\mathrm{H}$. bimaculata and $\mathrm{P}$. sphenops. Numerical method (No) and Frequency of occurrence method (F.O.)

\begin{tabular}{|c|c|c|}
\hline $\begin{array}{l}\text { Componentes } \\
\text { alimenticios }\end{array}$ & H. bimaculata & P. sphenops \\
\hline Oedogonium sp. & & $2.0(56.6)$ \\
\hline Spirogyra sp. & & $0.7(25.2)$ \\
\hline Cladophora fracta & & $0.02(3.9)$ \\
\hline Schizootrix sp. & & $2.0(5.1)$ \\
\hline Semillas & $0.03(0.6)$ & $0.2(10.3)$ \\
\hline Restos Vegetales & $0.01(0.6)$ & $1.8(69.0)$ \\
\hline Diptera & $7.0(12.0)$ & \\
\hline Diptera (Culicidae) & $9.8(23.2)$ & $0.5(17.1)$ \\
\hline Diptera (Simuliidae) & $6.3(11.0)$ & $1.9(9.7)$ \\
\hline Ephemeroptera & $6.7(12.0)$ & $0.9(8.4)$ \\
\hline Odonata & $9.3(19.9)$ & $0.05(10.2)$ \\
\hline Hymenoptera & $15.0(39.3)$ & \\
\hline Coleoptera & $10.3(24.5)$ & \\
\hline Hemiptera & $8.6(17.3)$ & \\
\hline Plecoptera & $6.9(11.7)$ & \\
\hline Collembola & $6.8(11.0)$ & \\
\hline Trichoptera & $6.2(9.0)$ & \\
\hline Arácnida & $1.2(2.6)$ & \\
\hline Micropterus salmoides & $4.1(6.2)$ & \\
\hline Huevos de Peces & $0.9(2.9)$ & \\
\hline Detrito & & $89.6(99.7)$ \\
\hline
\end{tabular}

Los valores están dados en porcentaje. (Los valores en paréntesis corresponden a la frecuencia).

Values in percentage. (The values in parenthesis are the frequencies).

obtuvieron una proporción de ingestión de 4.3 $\%$, registraron un valor de preferencia similar al de los arácnidos, por esta razón los dos fueron alimento circunstancial. El resto de los componentes alimenticios exhibieron valores relativamente bajos en ambas temporadas por lo que son registrados como alimentos circunstanciales o accidentales (Cuadro 2).

El análisis estacional de la dieta de $H$. bimaculata exhibió pequeñas diferencias, registrando que los alimentos accidentales en el período de lluvias (semillas y tricópteros) no fueron ingeridos en la temporada de estiaje, por otro lado, los peces (M. salmoides) únicamente se encontraron en la temporada de estiaje. A pesar de las diferencias que se registraron en la dieta por temporadas, el resultado de la prueba de $\chi^{2}$ demostró que las diferencias no son significativas $(p>0.05)$. Con base en el resultado obtenido con el índice de traslape de nicho, se observó que existe un traslape significativo entre ambas temporadas (0.93).

Análisis de la dieta por sexos: se estudiaron los tractos digestivos de 38 machos y 152 hembras y se detectó que estas últimas presentaron mayor diversidad de componentes alimenticios en su dieta (16), que los machos (11) (Cuadro 3). Esta diferencia puede ser producto a que el número de organismos analizados para cada sexo fue distinto, cabe mencionar que normalmente los poecílidos en la naturaleza presentan una proporción sexual sesgada hacia las hembras. En cuanto al análisis cuantitativo del alimento, se observó que no existen diferencias alimenticias entre sexos, puesto que ambos tienen valores de consumo relativamente similares. El orden Diptera exhibió las cifras más altas en los machos como alimento preferente y en las hembras como circunstancial, siendo los culícidos los más representativos, registrando en los machos los valores más altos. Los himenópteros también presentaron cifras altas, seguidos de los coleópteros y odonatos donde se reconoce que los machos alcanzan los valores de ingestión y preferencia mayores, el resto de los componentes alimenticios presentaron cifras relativamente bajas, considerados por esto como circunstanciales o accidentales.

Con base en los resultados con la prueba de $\chi^{2}$, se puede señalar que no existen diferencias alimenticias significativas entre sexos $(\mathrm{p}>0.05)$, lo cual fue corroborado con el resultado obtenido 
CUADRO 2

Dieta por épocas del año de H. bimaculata y P. sphenops, de acuerdo con los métodos numérico (No) y Frecuencia de Presencia (F.O.)

TABLE 2

Diet according to seasonal of $\mathrm{H}$. bimaculata and $\mathrm{P}$. sphenops. Numerical method (No) and Frequency of occurrence method (F.O.)

Componentes

alimenticios

Oedogonium sp.

Spirogyra sp.

Cladophora fracta

Schizootrix sp.

Semillas

Restos Vegetales

Diptera

Diptera (Culicidae)

Diptera (Simuliidae)

Ephemeroptera

Odonata

Hymenoptera

Coleoptera

Hemiptera

Plecoptera

Collembola

Trichoptera

Arachnida

Micropterus salmoides

Huevos de Peces

Detrito

H. bimaculata

Lluvias

Estiaje

Lluvias

Estiaje

\begin{tabular}{|c|c|}
\hline \multicolumn{2}{|l|}{$0.2(1.6)$} \\
\hline & $0.6(1.0)$ \\
\hline 8.9 (16.9) & $7.4(10.1)$ \\
\hline $9.4(25.4)$ & $9.9(22.8)$ \\
\hline $6.5(11.8)$ & $7.4(10.1)$ \\
\hline $7.3(11.8)$ & $7.4(10.1)$ \\
\hline $12.1(28.8)$ & 7.5 (19.6) \\
\hline $17.3(55.9)$ & $12.7(28.1)$ \\
\hline $7.3(11.8)$ & $11.0(27.4)$ \\
\hline $7.3(11.8)$ & $8.1(19.2)$ \\
\hline $6.4(11.8)$ & $7.4(10.1)$ \\
\hline $8.3(11.8)$ & $7.4(10.1)$ \\
\hline \multicolumn{2}{|l|}{$6.5(11.8)$} \\
\hline \multirow[t]{2}{*}{$0.9(3.3)$} & $0.6(1.8)$ \\
\hline & $4.3(9.4)$ \\
\hline 0.1 (1.6) & $1.4(3.7)$ \\
\hline
\end{tabular}

Los valores están dados en porcentaje. (Los valores en paréntesis corresponden a la frecuencia).

Values in percentage. (The values in parenthesis are the frequencies).

con el índice de traslape de nicho (0.93), lo que representa que existe un traslape.

Análisis de la dieta por tallas: con base en la longitud patrón, los ejemplares en forma arbitraria se agruparon en tres clases de talla, abarcando de 13 a $69 \mathrm{~mm}$, registrándose que la clase de talla II $(32-50 \mathrm{~mm})$ fue la que exhibió el número mayor de componentes alimenticios en su dieta de 14, mientras que la clase I (13$31 \mathrm{~mm})$ tuvo 12 y la III $(51-69 \mathrm{~mm}) 11$. Las proporciones de ingestión y preferencia en las tres clases presentaron al orden Diptera con los porcentajes de ingestión y preferencia mayores, registrándose en las tallas I y III como alimento preferente y en la talla II como circunstancial, 
CUADRO 3

Dieta de H. bimaculata $y$ P. sphenops por sexos, de acuerdo con los métodos numérico (No) y Frecuencia de Presencia (F.O.)

TABLE 3

Diet of $\mathrm{H}$. bimaculata and $\mathrm{P}$. sphenops according to sex. Numerical method (No) and Frequency of occurrence method (F.O.)

Componentes

H. bimaculata

P. sphenops

alimenticios

\begin{tabular}{|c|c|c|c|}
\hline \multicolumn{2}{|c|}{ H. bimaculata } & \multicolumn{2}{|c|}{ P. sphenops } \\
\hline Hembras & Machos & Hembras & Machos \\
\hline & & $2.0(53.9)$ & $2.0(62.4)$ \\
\hline & & $0.6(25.4)$ & $0.8(24.8)$ \\
\hline & & $0.06(4.5)$ & $0.03(2.8)$ \\
\hline & & $0.02(4.9)$ & $0.04(5.6)$ \\
\hline $0.03(0.6)$ & & $0.3(8.7)$ & $0.2(13.4)$ \\
\hline $0.01(0.6)$ & & $1.9(65.7)$ & $1.5(75.1)$ \\
\hline $7.2(12.6)$ & $8.3(13.4)$ & & \\
\hline $9.8(24.5)$ & $11.3(23.9)$ & $3.8(13.1)$ & $1.4(7.8)$ \\
\hline $6.4(10.7)$ & $7.8(13.4)$ & $2.3(4.7)$ & \\
\hline $6.9(10.0)$ & $7.9(16.0)$ & $1.5(2.8)$ & \\
\hline $9.0(20.5)$ & $11.4(21.3)$ & $2.7(5.1)$ & $0.7(2.8)$ \\
\hline $13.7(38.9)$ & $17.8(39.7)$ & & \\
\hline $9.5(21.8)$ & $12.6(29.2)$ & & \\
\hline $7.1(12.6)$ & $11.6(23.9)$ & & \\
\hline $6.8(11.3)$ & & & \\
\hline $7.4(10.7)$ & $7.7(13.4)$ & & \\
\hline $6.2(8.7)$ & & & \\
\hline $1.2(2.6)$ & & & \\
\hline $5.4(7.2)$ & $2.8(5.2)$ & & \\
\hline $1.7(3.2)$ & $0.2(2.6)$ & & \\
\hline & & 85.8 (99.6) & $93.0(100)$ \\
\hline
\end{tabular}

Oedogonium sp.

Spirogyra sp.

Cladophora fracta

Schizootrix sp.

Semillas

Restos Vegetales

Diptera

Diptera (Culicidae)

Diptera (Simuliidae)

Ephemeroptera

Odonata

Hymenoptera

Coleoptera

Hemiptera

Plecoptera

Collembola

Trichoptera

Arácnida

Micropterus salmoides

Huevos de Peces

Detrito

\section{(n)}

Componentes
alimenticios
Oedogonium sp.
Spirogyra sp.
Cladophora fracta
Schizootrix sp.
Semillas
Restos Vegetales
Diptera
Diptera (Culicidae)
Diptera (Simuliidae)
Ephemeroptera
Odonata
Hymenoptera
Coleoptera
Hemiptera
Plecoptera
Collembola
Trichoptera
Arácnida
Micropterus salmoides
Huevos de Peces
Detrito

Los valores están dados en porcentaje. (Los valores en paréntesis corresponden a la frecuencia).

Values in percentage. (The values in parenthesis are the frequencies).

observándose a la familia Culicidae con los porcentajes más altos en la talla II, en tanto que en la talla III los simúlidos fueron los que presentaron los valores mayores. Asimismo, los himenópteros exhibieron porcentajes altos en las tres clases de talla, considerándose como alimento preferente en las tallas I y III, y como circunstanciales en la talla II. Los peces $(M$. salmoides) fueron ingeridos solo en las clases I y II, exhibiendo en esta última un porcentaje relativamente alto (8.2), sin embargo, su preferencia fue sólo de $11.5 \%$, por lo que corresponde a un alimento secundario. El resto de los alimentos de origen animal presentaron cifras relativamente bajas, por lo que son estimados como alimentos circunstanciales o accidentales 
en las tres tallas. Las tallas II y III son las únicas que muestran ingesta de vegetales, donde se reconocieron semillas y restos vegetales con porcentajes bajos (Cuadro 4).

En cuanto al traslape entre las tres clases de talla, se obtuvieron valores altos 0.83 entre las tallas I y II, 0.74 entre las tallas I-III y de 0.79 entre las tallas II-III, valores que indican un alto grado de traslape entre tallas. Con base en el resultado de la prueba de $\chi^{2}$ se observó que las dietas por tallas no presentan diferencias significativas $(\mathrm{p}>0.05)$.

\section{P. sphenops}

Composición global de la dieta: la dieta de $P$. sphenops (404 ejemplares) estuvo constituida por 11 componentes alimenticios, siendo

\section{CUADRO 4}

Dieta de H. bimaculata y P. sphenops por clases de talla, de acuerdo con los métodos numérico (No) y Frecuencia de Presencia (F.O.)

TABLE 4

Diet $\mathrm{H}$. bimaculata and $\mathrm{P}$. sphenops according to size. Numerical method (No) and Frequency of occurrence method (F.O.)

\section{Componentes}

alimenticios

Oedogonium sp.

Spirogyra sp.

Cladophora fracta

Schizoothrix sp.

Semillas

Restos Vegetales

Diptera

Diptera (Culicidae)

Diptera (Simuliidae)

Ephemeroptera

Odonata

Hymenoptera

Coleoptera

Hemiptera

Plecoptera

Collembola

Trichoptera

Arachnida

Micropterus salmoides

Huevos de Peces

Detrito

\section{H. bimaculata}

I

II

III

$\begin{array}{ll} & \\ & \\ & \\ & 0.02(10.2) \\ & 7.2(10.2) \\ 11.0(29.9) & 10.0(24.9) \\ 7.9(29.9) & 7.4(10.2) \\ 12.3(35.4) & 8.4(17.5) \\ 18.1(53.1) & 11.5(33.3) \\ 12.2(35.4) & 11.8(24.9) \\ 7.9(23.1) & 7.2(10.2) \\ 9.8(25.8) & 7.6(11.2) \\ 7.4(21.7) & \\ 0.4(1.3) & 1.6(2.1) \\ 1.2(2.7) & 8.2(11.5) \\ 1.8(4.1) & 1.2(2.1)\end{array}$

P. sphenops

II III

$\begin{array}{lll}1.7(24.2) & 2.5(66.5) & 0.9(87.3) \\ 0.2(4.6) & 1.1(28.1) & 0.3(54.9) \\ & 0.02(2.4) & 0.2(14.1) \\ 0.01(0.7) & 0.02(4.4) & 0.04(14.1) \\ 0.8(9.3) & 0.05(9.3) & 0.2(15.5) \\ 3.0(39.0) & 1.4(83.2) & 0.9(83.1)\end{array}$

$12.2(39.2) \quad 7.4(25.9) \quad 0.2(5.6)$

$18.8(21.0) \quad 3.4(8.7) \quad 0.07(1.7)$

$11.4(16.4) \quad 1.7(5.6)$

$14.7(39.2) \quad 4.5(11.1)$

$19.1(57.3)$

$7.1(25.5)$

$7.8(21.0)$

$7.5(21.0)$

$0.2(4.5)$

$0.4(4.5)$

Los valores están dados en porcentaje. (Los valores en paréntesis corresponden a la frecuencia).

Values in percentage. (The values in parenthesis are the frequencies). 
el detrito el que alcanzó la proporciones más alta tanto en ingestión como en frecuencia ( $89.6 \%$ y $99.7 \%$, respectivamente). El resto de los alimentos registraron proporciones bajas, siendo considerados como alimentos circunstanciales (Cuadro 1).

Análisis temporal de la dieta: el detrito mostró, tanto en la estación de lluvias como en el estiaje, las proporciones de ingestión y FO más altas, por lo que fue considerado como alimento preferente, el resto de los componentes alimenticios son secundarios o circunstanciales debido a las cifras bajas alcanzadas (Cuadro 2). Los resultados demostraron que no existen diferencias significativas alimenticias por temporadas ( $p>0.05$ ), lo cual fue corroborado aplicando una prueba de $\chi^{2}$. Con base en el resultado obtenido con el índice de traslape de nicho, se observó que existe un traslape significativo entre ambas temporadas (0.99).

Análisis de la dieta por sexos: el análisis del tracto digestivo de 141 machos y 263 hembras, mostró que estas últimas presentan una mayor gama de componentes alimenticios (Cuadro 3). En cuanto al porcentaje de ingestión y preferencia, el detrito fue el que exhibió los valores más altos en ambos sexos. El resto de los componentes alimenticios muestran relativamente las mismas proporciones en ambos métodos en los dos sexos, por lo que no se observaron diferencias en la ingesta de alimentos. Con base en lo anterior, se puede apreciar que la dieta de ambos sexos se basa principalmente en el detrito, por lo que existe un alto traslape, lo cual fue corroborado con el resultado obtenido con el índice de traslape (0.99). Asimismo, el resultado de la prueba de $\chi^{2}$ demostró que no existen diferencias significativas $(\mathrm{p}>0.05)$.

Análisis de la dieta por tallas: con base en la longitud patrón, los organismos fueron agrupados en forma arbitraria en tres clases de talla que van de los 14 a los $88 \mathrm{~mm}$, donde la clase I varía de 14 a $34 \mathrm{~mm}$, la II de 35 a 65 $\mathrm{mm}$ y la clase III de 66 a $88 \mathrm{~mm}$. En las tres clases el detrito fue el que presentó los valores más altos de ingestión (Cuadro 4), alcanzando las proporciones mínimas en la talla I (76.8 \% ingestión y $99.2 \%$ frecuencia) y los porcentajes mayores en las clases de tallas II y III. En cuanto a preferencia alcanzaron el $100 \%$, por lo que fue estimado como alimento preferente en las tres clases de talla. El índice de traslape proporcionó valores de 0.99 para las tres clases de talla, lo cual indica que existe un traslape alto, a pesar que en la talla III no se apreciaron cuatro componentes alimenticios, pero este traslape es dado por el detrito, el cual presentó porcentajes de ingestión y preferencia altos en todas las talla. El resultado de la prueba de $\chi^{2}$ demostró que no existen diferencias significativas por clases de talla $(\mathrm{p}>0.05)$.

\section{Análisis interespecífico}

Los resultados de la dieta de cada una de las especies y su posterior comparación, mostró que existen diferencias alimenticias entre ellas (Cuadro 1). De esta forma $H$. bimaculata presenta mayor gama de componentes (16) en su dieta, mientras que $P$. sphenops únicamente registró once. La primera especie presentó un número mayor de alimentos de origen animal, en donde el orden Diptera fue el que exhibió los porcentajes de ingestión y de preferencia más altos, en tanto que $P$. sphenops tuvo un número mayor de componentes alimenticios vegetales, pero con porcentajes bajos de ingestión; asimismo, se observó la ingestión de detrito siendo el que mostró los valores máximos.

Se registró que estas especies sólo comparten seis alimentos (semillas, restos vegetales, odonatos, efemerópteros, dípteros culícidos y simúlidos) en bajas proporciones, por lo que se consideró que no existe traslape interespecífico, puesto que presentan dietas diferentes. Lo anterior fue corroborado con la aplicación del índice de traslape del que se obtuvo un valor de 0.02 .

\section{DISCUSIÓN}

Los resultados señalan que $H$. bimaculata consume una gama relativamente amplia de componentes alimenticios, la cantidad mayor 
de los ingeridos son de origen animal, principalmente insectos, de tal manera que a esta población se le debe ubicar como carnívora insectívora. Trujillo (1998) en un estudio realizado en el río Amacuzac, registró a esta especie como carnívora insectívora, observándose a los dípteros e himenópteros como componentes con porcentajes mayores al igual que en el presente estudio, y reconociendo que esta especie presenta una ingestión mayor de estos organismos. Asimismo, Hess y Tarzwell (1942) estudiaron la dieta del poecilido Gambusia affinis affinis y encontraron que se alimenta de diferentes grupos de origen animal, en donde los insectos son los que exhiben los valores más altos, razón por la cual estos autores también consideran a esta especie como carnívora-insectívora. Suárez et al. (2002) informan que la dieta de Gambusia punctata en el oriente de Cuba, está constituida por 34 alimentos exclusivamente de origen animal, en donde el porcentaje mayor lo registran los insectos acuáticos y terrestres $(95.5 \%)$, a pesar de lo anterior, los autores consideran a G. punctata como una especie generalista. No obstante, Gerking (1994) menciona que las especies generalistas no presentan una amplia preferencia por un recurso alimenticio en particular, asimismo, menciona que el término generalista no debería ser considerado como un esquema de clasificación alimenticia, por lo que G. punctata debe ser ubicada como carnívora-insectívora, al igual que $H$. bimaculata y G. affinis affinis.

En cuanto a los resultados en la población de $P$. sphenops, se observó que en comparación con $H$. bimaculata presenta una gama menor de componentes en su dieta, éstos son de origen vegetal, animal y detritos, siendo este último el que presentó los valores más altos de ingestión y preferencia, por lo tanto debe ser ubicado como un pez detritívoro. Martínez (1989) menciona que $P$. sphenops en el embalse Zicuirán, Michoacán, muestra hábitos alimentarios de tipo filtrador y ramoneador, debido a que su dieta está constituida por elementos del fitoplancton, así como de algas filamentosas del perifiton que contribuyen con una sexta parte de la dieta, también registró zooplancton (rotíferos y crustáceos) y un conjunto heterogéneo de organismos (protozoarios y larvas de insectos). Sin embargo, Torrente et al. (1993) en un estudio realizado en charcas permanentes y semipermanentes en Uraba al noroeste de Colombia, registraron que la dieta de $P$. sphenops, estaba constituida por gran cantidad de larvas de mosquitos, por lo cual consideran a esta especie como carnívora-insectívora.

Los resultados de Martínez (1989) y Torrente et al. (1993) parecen contradictorios con los obtenidos en el presente estudio, donde, como ya se mencionó, se concluye que $P$. sphenops es detritívora. Sin embargo, más que opuestos son complementarios, ya que su interpretación conjunta parece indicar que es una especie de alta plasticidad, dado que dispone del alimento presente en los diferentes ecosistemas que habita. Lo mismo ocurre con Poecilia reticulata que ha sido reportada como una especie insectívora (Machado-Allison 1987, Ortaz 1992). Ortaz (2001) la reporta como una especie herbívora ya que el $75 \%$ de su dieta está constituida por microalgas. En este sentido se ha mencionado que los poecílidos presentan flexibilidad alimentaria, dado que no sólo explotan diversos componentes alimenticios, sino también ocupan diferentes niveles tróficos. Esto se ha observado en Belonesox belizanus ubicada como una especie piscívora (Miley 1978, Turner y Snelson 1984); G. affinis como omnívora o carnívora (Hess y Tarzwell 1942, Greenfield et al. 1983a, b), Poecilia latipinna como herbívora (Harrington y Harrington 1961, 1982, Wetzel 1971) y G. punctata como generalista (Suárez et al. 2002)

Wikramanayake (1990) y Wootton (1992) afirmaron que la orientación y forma de la cavidad bucal son de alguna manera atributos relacionados con el comportamiento alimentario. Barrington (1957) y Meffe y Snelson (1989) mencionan que la dieta de los poecilidos es comúnmente reflejada por su morfología, dado que los dientes y la estructura del intestino son indicadores de las preferencias de la dieta. De esta manera, los dientes fuertes y cónicos, asociados a pequeños intestinos son típicos de especies depredadoras, características que en 
el presente estudio corresponden a H. bimaculata, las mismas peculiaridades han sido registradas en B. belizanus, G. affinis (Meffe et al. 1983, Turner y Snelson 1984). En cambio las especies omnívoras-detritívoras presentan un intestino largo y dientes espatulados, rasgos que fueron observados en $P$. sphenops en el presente estudio, y que han sido encontradas también en Poeciliopsis sp., reportada como especie detritívora (Vrijenhoek y Schultz 1974, Schoener 1981) y en Poeciliopsis occidentalis que es ramoneadora (Meffe 1984). Paredes (1998) menciona que los dientes espatulados son utilizados para extraer insectos y crustáceos entre las algas que cubren las rocas y para raspar algas.

Schoener (1982) menciona que la alimentación de los peces puede variar de acuerdo con la localidad y época del año y las variaciones en la dieta pueden estar relacionadas con la disponibilidad de alimento en un momento dado. En el presente estudio, se registró que la dieta de $H$. bimaculata exhibió pequeñas diferencias en el porcentaje de ingestión de los alimentos consumidos, observándose que las semillas y tricópteros no fueron ingeridos en la temporada de estiaje y los himenópteros obtuvieron un porcentaje mayor en las lluvias. Por otro lado, los peces (M. salmoides) únicamente se encontraron en la temporada de estiaje. Sin embargo, estas diferencias no son consideradas como significativas, ya que se observó que la dieta primordial en ambas temporadas fueron los dípteros e himenópteros. Asimismo, se registró que $P$. sphenops no mostró diferencias estacionales significativas en la dieta, puesto que en la época de lluvias como en el estiaje basa su alimentación en el detritos. Ortaz (2001) informó que $P$. reticulata en el río Orituco al norte de Venezuela no presentó diferencias alimenticias por temporadas.

El análisis de la dieta por sexos demostró, con la excepción del número de alimentos que ingieren, que ninguna de las dos especies mostraron diferencias. Duarte (1981) realizó un estudio con ocho especies de peces de la familia Goodeidae y menciona que no se observaron diferencias alimenticias sexuales. En cambio Hess y Tarzwell (1942) observaron que G. affinis affinis tiene diferencias alimenticias por sexos y estas diferencias están dadas por la talla de los peces. Así, los machos comen sólo alrededor de la mitad de los componentes que ingieren las hembras de la misma longitud, también encontraron que el alimento en las hembras se incrementó conforme ganaban talla, consumiendo altos porcentajes de organismos macroscópicos, principalmente larvas del mosquito Anopheles sp.

En forma semejante a los análisis por sexos, la dieta de $P$. sphenops y $H$. bimaculata no tuvo variaciones significativas cuando se analizó en relación con la talla; tanto los peces pequeños como los grandes mostraron preferencia por los mismos componentes alimenticios. Martínez (1989) analizó los hábitos alimentarios de $P$. sphenops en el embalse michoacano Zicuirán y al igual que en el presente estudio, no encontró diferencias relacionadas con la talla, debido a que muestran preferencia por los mismos alimentos. Trujillo-Jiménez y Díaz-Pardo (1996), mencionan que Ilyodon whitei tiene diferencias alimenticias ontogénicas, puesto que los jóvenes mostraron preferencias principalmente por el alimento de origen animal, en tanto que los adultos lo hacen por los vegetales.

Con base en el análisis de la dieta de ambas especies se registró que no existe traslape trófico entre éstas, puesto que son diferentes, observándose que $H$. bimaculata muestra una gama de componentes alimenticios donde los insectos fueron el alimento principal; mientras que $P$. sphenops presenta una cantidad menor de alimentos en su dieta donde el detrito fue el preferente y el resto de los alimentos registrados muestran porcentajes bajos. Esto puede ser debido a que existe una cierta segregación espacial dentro de la columna de agua, ya que $P$. sphenops se alimenta principalmente en la parte media y profunda de la columna (Zaret y Rand 1971), mientras que H. bimaculata se alimenta en la superficie. Un resultado semejante es informado por Meffe et al. (1983), quienes determinaron que $P$. occidentalis y $G$. affinis presentaron escaso traslape en la dieta, pero observaron que el alimento es abundante en 
los ecosistemas donde habitan estas especies (varias localidades en el sureste de Arizona). Sato (1989) observó que G. affinis y Oryzias latipes compiten por el alimento, principalmente por las larvas de mosquitos, sin embargo, la primera presenta una ingestión mayor de estas larvas y además en su dieta incluye individuos de la segunda especie, ocasionando que la densidad de población de $O$. latipes disminuya.

Los resultados anteriores permiten percatarse que los peces como lo menciona Wooton (1991), presentan gran plasticidad en la ecología alimenticia, ya que tienen la capacidad de ocupar distintos niveles tróficos, que van desde las especies herbívoras hasta las carnívoras secundarias e incluso algunas especies aprovechan la materia orgánica en descomposición. Es decir, utilizan el detrito como alimento, tal como se muestra en el presente estudio, donde $P$. sphenops es considerada detritívora mientras que $H$. bimaculata es ubicada como carnívora o insectívora.

Resulta claro, que no se debe generalizar en cuanto a la dieta de los peces, como lo menciona Nikolskii (1969) esta dieta puede variar desde el punto de vista ontogénico, estacional y sexual. Algunas de estas variaciones se deben principalmente a las diferencias morfológicas, fisiológicas y conductuales de los organismos, así como a la disponibilidad del alimento, tanto en abundancia como en diversidad. Esto se observó en los diferentes trabajos que se discutieron en el presente estudio, donde se registraron variaciones de la dieta de diferentes especies de acuerdo conl sexo, talla y estacionalidad.

\section{AGRADECIMIENTOS}

Agradecemos a Edmundo Díaz Pardo, Héctor Quiroz Castelán, Isela Molina Astudillo, Juan Carlos Sandoval Manrique, Joel Paulo Maya, Eugenia López López, Fausto Méndez de la Cruz y Julio Camargo Benjumeda por la revisión critica del manuscrito.

\section{RESUMEN}

Se analizó la dieta y los hábitos alimentarios de Heterandria bimaculata y Poecilia sphenops. Se tomaron muestras mensuales en la presa "Los Carros", Morelos, México $\left(10^{\circ} 37^{\prime} \mathrm{N}, 98^{\circ} 43^{\prime} \mathrm{W}\right)$. El contenido intestinal fue analizado de acuerdo con los métodos numérico (No) y el de frecuencia de presencia (FO). Se utilizó el índice de traslape de MacArthur y Levin. H. bimaculata presentó la ingestión de 16 componentes alimenticios. Los odonatos y los himenópteros fueron los más consumidos en le estación de lluvias, mientras que en el estiaje fueron los himenópteros y coleópteros. La talla II fue la que registró la mayor diversidad, siendo los himenópteros los más consumidos en las tres tallas. El índice de traslape en cada uno de los análisis fue de 0.74 a 0.99 . P. sphenops consumió 11 tipos de alimento; el detrito registró los porcentajes más altos independientemente de sexo, tamaño y época (índice de traslape $=0.99$ ). Ambas especies comparten seis alimentos, pero en bajas proporciones (índice de traslape $=0.26$ ).

Palabras clave: dieta, hábitos alimentarios, Heterandria bimaculata, Poecilia sphenops, "Los Carros", Morelos, México.

\section{REFERENCIAS}

Alayo, P. 1973. Lista de peces fluviales de Cuba. Torreia, Nueva Serie 29:1-59.

Álvarez del Villar, J. 1970. Peces Mexicanos (Claves) México. Serie Investigaciones Pesqueras, Instituto Nacional de Investigaciones Biológico Pesqueras, México, DF, México. 166 p.

Barrington, E.J.W. 1957. The alimentary canal and digestion, p. 109-161. In W.S. Hoar \& D.J. Randall (eds.). Fish physiology. Academic, Nueva York, EEUU.

Bland, R.G. 1979. How to know the insects. WMC Brown, Iowa, EEUU. 409 p.

Chu, H.F. 1979. How to know. The inmature insects. WMC Brown, Iowa, EEUU. 243 p.

Duarte, S.M.P. 1981. Contribución al conocimiento de los hábitos alimentarios de doce especies de peces en el lago de Cuitzeo, Michoacán. Tesis de Licenciatura, Instituto Politécnico Nacional, México, D.F., México. 45 p. 
Gerking, D.S. 1994. Feeding ecology of fish. Academic, Londres, Inglaterra. 416 p.

Greenfield, D.W., T.A. Greenfield \& L. Brinton. 1983a. Spatial and trophic interaction between Gambusia sexradiata and Gambusia puncticulata yucatana (Pisces: Poeciliidae) in Belize, Central America. Copeia 3: 598-607.

Greenfield, D.W., C.F. Rakocinski \& T.A. Greenfield. 1983b. Spatial and trophic interaction in wet and dry season between Gambusia luma and Gambusia sexradiata (Pisces: Poeciliidae) in Belize, Central America. Fieldiana Zool. 14: 1-16.

Harrington, R.W., Jr. \& E.S. Harrington. 1961. Food selection among fishes invading a high subtropical salt marsh: from on set of flooding through the progress of a mosquito brood. Ecol. 42: 649-652.

Harrington, R.W., Jr. \& E.S. Harrington. 1982. Effects on fishes and their forage organisms of impounding a Florida marsh to prevent by salt marsh mosquitoes. Bull. Mar. Sci. 32: 523-31.

Hess, A.D. \& C.M. Tarzwell. 1942. The feeding habits of Gambusia affinis affinis, with special reference to the malaria mosquito, Anopheles quadrimaculatus. Am. J. Hygiene 1:142-151.

Krebs, J.C. 1999. Ecological methodology. HarperCollins, Nueva York, EEUU. 654 p.

Lagler, K.F. 1956. Freshwater fishery biology. WMC Brown, Iowa, EEUU. 421 p.

Magallón-Barajas, S., J. Paulo-Mayo \& E. Díaz-Pardo. 1992. Avances en el conocimiento de la presa "Los Carros" Morelos, un embalse de reciente formación. Univ. Cienc. Tecnol. 2: 121-129.

Machado-Allison, A. 1987. Los peces de los llanos de Venezuela: un ensayo sobre su historia natural. Universidad Central de Venezuela, Caracas, Venezuela. 143 p.

Martínez, T.M. 1989. Contribución al conocimiento de la biología de Poecilia sphenops Valenciennes (Pisces: Poecilidae) en la presa Zicuirán, Michoacán. Bol. Univ. Michoacana de San Nicolás de Hidalgo 2: 49-63.

Merrit, W.R. \& K.W. Cummins. 1996. An introduction to the aquatic insects of the North America. Kendall Hunt, Iowa, EEUU. 862 p.
Meffe, G.K. 1984. Density-dependent cannibalism in the endangered Sonoran Topminnow (Poeciliopsis occidentalis). Southwestern Nat. 29: 500-503.

Meffe, G. \& F. Snelson. 1989. Ecology and evolution of livebearing fishes (Poeciliidae). Prentice Hall, Nueva Jersey, EEUU. 453 p.

Meffe, G.K., D.A. Hendrickson \& W.L. Mincley. 1983. Factors resulting in decline of the endangered Sonora topminnow Poeciliopsis occidentalis (Atheriniformes: Poeciliidae) in the United States. Biol. Conserv. 25: 135-159.

Miley, W.W. 1978. Ecological impact of the pike killifish, Belonesox belizanus Kner, (Poeciliidae) in southern Florida. Tesis de Maestría, University of Florida, Florida, EEUU. 88 p.

Moyle, B.P. \& J.J. Cech, Jr. 1988. Fishes an introduction to ichthyology. Prentice Hall, Nueva Jersey, EEUU. $559 \mathrm{p}$.

Needham, J.G. \& P.R. Needham. 1978. Guía para el estudio de los seres vivos de las aguas dulces. Reverté, Barcelona, España. 131 p.

Nikolskii, G.V. 1969. Theory of fish population dynamics as the biological background for rational exploitation and management of fishery resources. Oliver and Boyd, Londres, Inglaterra. 326 p.

Ortaz, M. 1992. Hábitos alimenticios de los peces de un río de montaña Neotropical. Biotropica 24: 550-559.

Ortaz, M. 2001. Diet seasonality and food overlap among fishes of the upper Orituco stream, northern Venezuela. Rev. Biol. Trop. 49: 191-197.

Paredes, L.M.E. 1998. Estudio ecomorfológico de Poecilia sphenops (Pisces: Cyprinodontiformes). Tesis de Licenciatura, Universidad Autónoma del Estado de Morelos, Cuernavaca, México. 42 p.

Parenti, R.L. \& M. Rauchenberger. 1989. Systematic Overview of the Poeciliines, p. 3-31. In G.K. Meffe \& F.F. Snelson Jr. (eds.). Ecology and Evolution of Livebearing Fishes (Poeciliidae). Prentice Hall, Nueva Jersey, EEUU.

Pennak, K. 1978. Freshwater invertebrate of the United States. Wiley, Nueva York, EEUU. 420 p.

Sato, H. 1989. Ecological studies on the mosquito fish Gambusia affinis for encephalitis control with special 
reference to selective feeding on mosquito larvae and competition with the medaka, Oryzias latipes. Japanese J. Trop. Med. Hyg. 2: 157-173.

Schoener, A.A. 1981. The role of competition in the displacement of native fishes by introduced species, $p$. 173-203. In R.J. Naiman \& D.L. Soltz (eds.). Fishes in North American Deserts. Wiley, Nueva York, EEUU.

Schoener, W.T. 1982. The controversy over interespecific competition. Am. Scient. 70: 586-595.

Siegel, S. 1976. Estadística no paramétrica. Trillas, México DF, México. 346 p.

Suárez, T S.I., D.G. Lambert; G.G. Garcés \& A. Fong. 2002. Algunos datos acerca de la alimentación de Gambusia punctata (Cyprinodontiformes: Poeciliidae) en una localidad del oriente de Cuba. AvaCient 33: 56-68.

Torrente, A., W. Roja, A. Uran, T. Kano \& S. Orduz. 1993. Fish species from mosquito breeding ponds in northwestern Colombia: evaluation of feeding habitats and distribution. Memorias do Instituto Oswaldo Cruz 4: 625-627.

Trujillo, J.P. 1998. Dinámica trófica de la ictiofauna del río Amacuzac, Morelos, México. Tesis de Maestría, Universidad Nacional Autónoma de México, México DF, México. 101 p.

Trujillo-Jiménez, P. \& E. Díaz-Pardo. 1996. Espectro trófico de Ilyodon whitei (Pisces: Goodeidae) en el río del muerto, Morelos, México. Rev. Biol. Trop. 44: 755-761.

Turner, J.S. \& F.F. Snelson Jr. 1984. Population structure, reproduction and laboratory behavior of the introduced Belonesox belizanus (Poeciliidae) in Florida. Env. Biol. Fish. 10: 89-100.

Vrijenhoek, R.C. \& R.J. Shultz. 1974. Evolution of a trihybrid unisexual fish (Poeciliopsis: Poeciliidae). Evolut. 28: 306-19.

Wetzel, R.L. 1971. Analysis of cohabitation by Gambusia affinis and Poecilia latipinna (Pisces: Poeciliidae) in a salt-marsh canal in Florida. Master Dissertation, University of West Florida, Pensacola, EEUU. 92 p.

Wikramanayake, D.E. 1990. Ecomorphology and biogeography of a tropical stream fish assemblage: evolution of assemblage structure. Ecol. 7: 1756-1764.

Windell, J.T. \& H. Bowen. 1978. Study of fish based on analysis of stomach contests, p. 219-226. In T. Bagenal (ed.). Methods for assessment of fish production in freshwater. Blackwell, Londres, Inglaterra.

Wooton, R.J. 1991. Ecology of teleost fishes. Fish and fisheries. Chapman \& Hall, Nueva York, EEUU. 404 p.

Wootton, R.J. 1992. Fish Ecology. Chapman \& Hall, Nueva York, EEUU. 221 p.

Zaret, T.M. \& A.S. Rand. 1971. Competition in tropical stream fishes: Support for the competitive exclusion principle. Ecol. 52: 336-342. 
